\title{
Una paz insostenible: abusos y embrollos discursivos en el uso del término "sostenible" aplicado a la idea de desarrollo
}

An Unsustainable Peace: Abuses and Discursive Muddles in the Use of the Expression "Sustainable" as Applied to the Idea of Development

José María Enríquez Sánchez Universidad de Valladolid, España https://orcid.org/0000-0003-1597-9250

Artículo de investigación

Fecha de recepción: 25 de febrero de 2020 Fecha de aceptación: 3 de abril de 2020

\section{Para citar este artículo:}

Enríquez Sánchez, J. M. (2020). Una paz insostenible: abusos y embrollos discursivos en el uso del término "sostenible" aplicado a la idea de desarrollo. Campos en Ciencias Sociales, 8(2), 159-185. Dor: https://doi.org/10.15332/25006681/6016

Doctor en Filosofía por la Universidad de Valladolid y doctor cum laude en Historia por la Universidad de Salamanca, con premio extraordinario de doctorado. Investigador-docente en la Universidad de Valladolid. Correo electrónico: josemaria.enriquez@uva.es 


\title{
RESUMEN
}

El presente escrito, como anticipa su título, versa sobre la idea de desarrollo sostenible y su falta de concreción. Una inconcreción debida al uso verborreico que se le ha ido dando a dicha expresión, lo que ha favorecido el hecho de que originariamente su contenido fuera inespecífico y, por ende, inútil para evitar los males del desarrollismo y sus efectos sobre la sociedad. De ahí la proliferación de planteamientos que, al no tratarse de críticas radicales, tampoco son capaces de mostrarse como alternativas a la argumentación oficial. Esto debido a una falta de atención a los presupuestos fundamentales que lo constituyen, como mostraremos a partir de un análisis crítico del discurso de las fuentes originarias —principalmente resoluciones de la Asamblea General y otros documentos de las Naciones Unidas - respecto del tema que aquí nos ocupará en lo sucesivo, hasta centrar la atención en asuntos menos especulativos y sí más obligados a acciones directas de respeto y cuidado hacia las personas y sus circunstancias como presupuestos para la concordia.

Palabras clave: colonización, imperialismo, desarrollo, sostenibilidad, descolonización del imaginario, paz imperfecta.

\begin{abstract}
As suggested by its title, this paper deals with the idea of sustainable development and its lack of concreteness. Such a lack of concreteness is due to the verbose use given to the expression "sustainable", which in turn has been favored by the fact that its content is originally nonspecific and, therefore, useless to avoid the evils of developmentalism and its effects on society. Hence the proliferation of approaches which, since they are not radical criticisms, are also not capable of revealing themselves as alternatives to official arguments, due to a lack of attention to the fundamental assumptions that constitute them, as we will show from a critical analysis of the discourse of the original sources on the subject (mainly resolutions of the General Assembly and other United Nations documents), before focusing our attention on matters that are less speculative and more committed to direct actions of respect and care for people and their circumstances as preconditions for harmony.
\end{abstract}

Keywords: colonization, imperialism, development, sustainability, decolonization of the imaginary, imperfect peace. 


\section{INTRODUCCIÓN: LA ADJETIVACIÓN SOSTENIBLE Y SU APLICACIÓN EN EL DISCURSO SOBRE EL DESARROLLO}

En primer lugar debemos precisar cómo ubicar el uso de la expresión sostenible en los discursos sobre el desarrollo, para lo cual consideramos que un buen acercamiento a ello nos lo proporciona un autor como Arrigo Coen Anitúa, quien nos aclara, tras un concienzudo análisis morfológico, lexicológico y semántico, que "sostenible se refiere al aspecto endoestructural del sistema de que se trate, lo que ha de permanecer firmemente establecido, asentado, fijo, inalterable, inamovible", mientras que:

[...] sustentable será lo supra- o superestructural de ese mismo sistema, lo que requiere que se lo esté alimentando, proporcionándole los medios de sobrevivencia y de persistencia, a fin de que pueda extender su acción, no solo en su ámbito (espacio) sino también en el tiempo. (Coen Anitúa 2006, pp. 50-51)

Aclarado este particular aspecto, se entenderá que en el tema que nos ocupa nosotros nos refiramos a la expresión sostenible. No obstante, ocurre que la palabra ha sido usada en contextos tan variopintos que en los últimos tiempos parece haber perdido verdadera significatividad, hasta un extremo tal que su sola alusión presume que nos hallamos ante un discurso bienintencionado. $\mathrm{O}$ sea, la sola expresión sostenibilidad — esto es, la cualidad de lo sostenible — se confunde con una consideración más valorativa que descriptiva, pero ¿es correcta esa equiparación? Ciertamente no y ello se explica de manera sencilla al constatar que no veríamos como bueno que un proceso morboso se alargara en el tiempo, ni que en el paciente se sostenga su pesadumbre; sin embargo, todo lo contrario, es lo que solemos esperar del sentimiento de amor hacia la pareja y de la lealtad a un amigo. Por eso decimos que la sostenibilidad no es de por sí un valor, como a veces parece colegirse de ciertos usos oportunistas en todo tipo de discursos que pretenden congraciarse con el público al que se dirige. Empero, algo nos ha quedado claro a partir de estas sencillas ejemplificaciones con las que pretendemos introducirnos en el tema que aquí nos ocupa: desde una perspectiva en exceso simplificada se podría fácilmente convenir en que el adjetivo sostenibilidad expresa la cualidad de un sistema que se puede mantener durante largo tiempo; pero para tratar de hacerla más operativa respecto a los propósitos de este escrito sería 
provechoso acotar su sentido, tal y como pretendieron Robert Costanza y Bernard C. Patten, quienes, al comienzo de su artículo Defining and predicting sustainability, lo plantearon en torno a tres cuestiones: 1) qué sistema, subsistemas o características de estos se pretenden preservar; 2) por cuánto tiempo se deben mantener esas condiciones; y 3) en qué momento evaluaremos si ese sistema, subsistemas o características han perdurado (1995, pp. 193-196).

Pues bien, un sistema económico será sostenible cuando no sufra grandes disrupciones que pongan en serio cuestionamiento sus principios; uno social lo será cuando sus transiciones a mejores condiciones de vida no solo no supongan una ruptura brusca con valores sociales anteriores, sino que conserve e impulse todos aquellos que son promovedores de lo social; y de manera similar, podemos hablar de sostenibilidad de un sistema biológico cuando se mantienen las condiciones para la vida y la reproducción de las especies.

Así, pues, lo que a partir de aquí sostendremos es que todos esos sistemas (económico, social y ecológico) no cumplen con las condiciones suficientes para su sostenibilidad. Razones para ello pueden argüirse bastantes dependiendo de cómo queramos iniciar este relato; pero, por nuestra parte, lo derivaremos en atención a los problemas del desarrollo y su incidencia sobre el deterioro medioambiental.

\section{LA NOCIÓN DE DESARROLLO Y SUS ADJETIVACIONES}

Como ocurre con el término anterior, no es menor la dificultad de explicitar qué se entiende por desarrollo y más aún si a ello habremos de adjetivarle después la cualidad de sostenible, pues pudiera ser el caso que las ventajas iniciales que se le atribuyen según la concepción de desarrollo desde una perspectiva cortoplacista no fueran aplicables en un margen temporal más largo, como se ha evidenciado desde los años setenta del pasado siglo $\mathrm{xx}$, cuando los problemas medioambientales derivados del desarrollismo alcanzaron tal magnitud que han llegado a poner en cuestión, incluso, la propia idea de progreso, que mucho le debe a los planteamientos historicistas. 
Pues bien, en el ámbito sociopolítico hablamos de desarrollo como un proceso de mejora por medio del cual se da cuenta de un determinado avance en la prosecución de un objetivo inequívoco de bienestar. En la medida en que esta prosperidad depende de las posibilidades de consumo, no es de extrañar que la principal concepción sobre el desarrollo se halle signada por indicadores de índole económico —como en el caso de producto interior bruto- - O al menos así ha sido tratado por buena parte de los teóricos de la economía del desarrollo, cuyas ideas llegaron a configurar la concepción predominante del desarrollo como una superación de etapas que culmina con el establecimiento de la sociedad de consumo de masas, que se presumía que era el tipo de sociedad ideal al que debían atender las políticas internacionales de desarrollo y que, por lo tanto, para estos países supondría un intento mimético por igualarse a aquellos otros, de ese modo aventajados. Para esto la consigna se expresó sin ambages: el abandono de las filosofías antiguas, la desintegración de las viejas estructuras sociales, la ruptura de los lazos de casta, credo y raza. Así lo propuso un grupo de expertos nombrado por Trygve Halvdan Lie, a la sazón primer Secretario General de las Naciones Unidas.

A partir de aquí (United Nations Publication n. ${ }^{\circ}$ 1951.ILB.2, chapter III, $\$ 36$ ), la habilidad de estas otras gentes para definir y hacerse cargo de sus propias vidas fue desgastada en favor de un único paradigma de desarrollo impulsado mediante diversos planes desde la Organización de las Naciones Unidas. Su intervencionismo encontró oportuna justificación en el capítulo Ix, artículo 55.a de la Carta de San Francisco, firmada el 26 de junio de 1945, en la que los Estados firmantes se comprometieron a promover "niveles de vida más elevados".

De este propósito se haría eco la resolución del 4 diciembre de 1948 de la Asamblea General de las Naciones Unidas sobre Desarrollo económico de los países insuficientemente desarrollados, en la que se consideró que estos niveles bajos de vida:

[...] entrañan consecuencias económicas y socialmente perniciosas para los países directamente interesados y para el mundo entero y producen una inestabilidad que es perjudicial para el mantenimiento de relaciones pacíficas 
y amistosas entre las naciones y para el desarrollo de las condiciones necesarias para el progreso económico y social. (A/RES/198[III])

De ahí la llamada al Consejo Económico y Social y los organismos especializados a tomar medidas que fomentaran el progreso económico y elevaran los niveles de vida de aquellos los países a los que no se dudaría en calificar de subdesarrollados.

Hasta entonces las relaciones con aquellos países habían sido las propias de los abusos colonizadores: la asimilación por conquista. Sin embargo, con la dicotomía que se establecía en estos nuevos tiempos, se hacía omisión de buena parte de las condiciones que habían favorecido esa insuficiencia, quedando como única respuesta a este imputado retraso la falta de asistencia y formación técnica capaces de promover una mayor producción y, con ello, favorecer el aumento de los niveles de vida.

Para este propósito, durante los años siguientes se fue creando todo un aparataje técnico y burocrático que hiciera posible esa asistencia para el desarrollo; pero también una construcción teórica por parte de no pocos autores, como Clarence Edwin Ayres, Paul Narcyz Rosenstein-Rodan, Kurt Mandelbaum, Ragnar Nurkse, Simon Kuznets, William Arthur Lewis, Berthold Frank Hoselitz o Walt Whitman Rostow, cuyas ideas sobre la modernización servirían de base para aquellas políticas, configurando una serie de temas que se harían característicos de lo que se daría en llamar economía del desarrollo y cuyo estudio de las condiciones del progreso comenzó a vincularse con la idea de bienestar.

Dicha posibilidad se establecía a partir de la idea de bienestar económico, con un aproximado objetivo monetario (Pigou, 2016, $\$ \$ 1-11$ ). En línea con esta tendencia, la denominada economía del desarrollo redujo toda idea de prosperidad a la del aumento de la renta nacional en la presunción de que cuanto mayor fuera la riqueza monetaria de un país, mayor sería el bienestar de sus gentes.

Este es todo el plan de desarrollo que a partir de entonces se promovería en sucesivos decenios desde la onu: emulación de los modos de vida y consumo de las sociedades occidentales. No obstante, las críticas a esta única visión del desarrollo y sus 
continuadas adjetivaciones como única respuesta, no hicieron variar la consideración sobre la idea de progreso. Se habló así de desarrollo autocentrado, endógeno, participativo, comunitario, social, local, integrado, auténtico, autónomo, popular, humano o equitativo, pero sin que ninguno de esos añadidos supusiera una verdadera reconsideración sobre los presupuestos que fundamentan la idea de desarrollo, incluida la actual que se ha adjetivado como sostenible, de la que partíamos y que desde aquí explicitaremos para mostrar, por un lado, la insuficiencia de la respuesta, pero también, por otro, la falta de alternativas que hasta la fecha no han hecho sino abundar en desatinos similares, precisamente por una completa desatención a las cuestiones de fondo, que, para sorpresa de muchos, son de tipo onto-gnoseológicos.

\section{EL SENTIDO ORIGINARIO DE LA EXPRESIÓN DESARROLLO SOSTENIBLE}

Resulta habitual en la literatura académica a este respecto remontar el origen de la expresión desarrollo sostenible al año de 1980. Por aquel entonces un estudio realizado por la Unión Internacional para la Conservación de la Naturaleza (UICN), con la asesoría, cooperación y apoyo financiero del Programa de las Naciones Unidas para el Desarrollo (PNUD) y el Fondo Mundial para la Naturaleza (WwF, por sus siglas en inglés: World Wildlife Fund), en colaboración con la Organización de las Naciones Unidas para la Agricultura y la Alimentación (FAO) y la Organización de las Naciones Unidas para la Educación, la Ciencia y la Cultura (Unesco), que llevaba por título Estrategia Mundial para la Conservación (EMC), introdujo, por vez primera, la noción de desarrollo sostenido en los siguientes términos argumentativos:

[...] las actividades humanas van reduciendo paulatinamente la capacidad que tiene nuestro planeta de mantener la vida, en una época en la cual el aumento de la población y del consumo plantea unas exigencias crecientes a dicha capacidad. El impacto destructor combinado de aquella mayoría de seres humanos pobres que luchan por subsistir, y de la minoría rica que consume la mayor parte de los recursos del globo, está socavando los medios que permitirían a todos los pueblos sobrevivir y florecer. (UICN/PNUMA/wwF, 1980 , cap. $1, \$ 1)$ 
De ahí surge la propuesta de integración de la conservación y del desarrollo, ya que — prosigue aquel texto— "si no se aplica en todas partes una estructura de desarrollo que conserve los recursos vivos, será imposible satisfacer las necesidades de hoy sin impedir lo que es preciso lograr mañana” (cap. $1, \$ 8$ ).

Este vínculo entre el desarrollo y la conservación, que constituye el sentido de la expresión desarrollo sostenido, no volvió a ser reiterado por ninguno de los documentos oficiales de las Naciones Unidas, hasta que en 1987 la Comisión para el Desarrollo y el Medio Ambiente (CDMA) presentara el informe Nuestro futuro común (también conocido como Informe Brundtland), en el que se trató de dar un nuevo enfoque a las políticas sobre desarrollo, distinguiendo entre fracasos del desarrollo y de la gestión del medio ambiente humano.

En dicho informe, respecto del primero de los fracasos se afirma que.

[...] en cifras absolutas, hay en el mundo más hambrientos que nunca anteriormente, y su número sigue aumentado. Al igual que el número de quienes no saben leer ni escribir, el número de los que carecen de agua limpia o de viviendas seguras y adecuadas y el número de los que sufren de escasez de leña para cocinar y protegerse del frío. La brecha que separa a las naciones ricas de las pobres se agranda en vez de achicarse y, dadas las tendencias y los arreglos institucionales presentes, son escasas las perspectivas de que el proceso cambie diametralmente de dirección. (A/42/427, I, 1, \$\$ 5-10)

De seguido, respecto de la mala gestión medioambiental, a partir de estudios previos, se señaló que el empeoramiento de las condiciones medioambientales "amenazan la vida de muchas de sus especies, incluida la humana” (A/42/427, I, 1, \$\$5-10).

Esto lleva a los autores de dicho informe a su propuesta de "hacer que el desarrollo sea sostenible, duradero, o sea, asegurar que se satisfaga las necesidades del presente sin comprometer la capacidad de las futuras generaciones para satisfacer las propias" (A/42/427, I, 3, \$27; cap. 1 , II, $\$ 49$ y cap. $2, \$ 1$ ss.). 
Pareciera que a tenor de lo leído, la expresión desarrollo duradero (planteada por la Comisión Brundtland) es parecida de la de desarrollo sostenido (propuesto por la UICN); sin embargo, la continuación del citado texto podría mostrar las desemejanzas:

El concepto de desarrollo duradero implica límites - no límites absolutos, sino limitaciones que imponen a los recursos del medio ambiente el estado actual de la tecnología y de la organización social y la capacidad de la biósfera de absorber los efectos de las actividades humanas. Pero tanto la tecnología como la organización social pueden ser ordenadas y mejoradas de manera que abran el camino a una nueva era de crecimiento económico. La Comisión cree que ya no es inevitable la pobreza general. La pobreza es no solo un mal en sí misma. El desarrollo duradero exige que se satisfagan las necesidades básicas de todos y que se extienda a todos la oportunidad de colmar sus aspiraciones a una vida mejor. Un mundo donde la pobreza es endémica estará siempre propenso a ser víctima de la catástrofe ecológica o de otro tipo. (A/42/427, I, $3, \S 27)$

A partir de esta lectura puede resultar fácil colegir que persista una culpabilización de la pobreza por el deterioro medioambiental. Sin embargo, esto se debe a una mala (y desafortunada) síntesis del contenido de aquel informe, pues en su parte expositiva la presumible culpabilidad de la pobreza quedaría anulada con el contenido expuesto en el siguiente fragmento:

Las fallas que necesitamos corregir se originan tanto en la pobreza como en la manera miope con que, a menudo, hemos tratado de conseguir la prosperidad. Muchas partes del mundo están enredadas en una maligna espiral descendente: los pueblos pobres se ven obligados a utilizar en exceso los recursos del medio ambiente para sobrevivir de día en día, y el empobrecimiento de su medio ambiente contribuye a acentuar su indigencia, y a hacer aún más difícil e incierta su supervivencia. La riqueza alcanzada en algunas partes del mundo es, a menudo, precaria porque se ha conseguido por prácticas agrícolas, forestales e industriales que proporcionan ganancias y progreso solo a corto plazo. $(\mathrm{A} / 42 / 427, \$ 3)$ 
Pero lo mismo que afirma esto respecto del comportamiento de los pobres, del de los ricos se dice:

Por otra parte, donde el crecimiento económico ha producido mejoras en los niveles de vida, algunas veces se ha conseguido en una forma que es perjudicial a más largo plazo. En el pasado, gran parte de las mejoras se basaron en la utilización cada vez en mayor escala de materias primas, energía, productos químicos y sintéticos, creando una contaminación que no se ha contabilizado suficientemente al calcular los costos de los procesos de producción. Estas tendencias han tenido efectos insospechados sobre el medio ambiente. De ahí que los desafíos al medio ambiente provienen tanto de la falta de desarrollo como de las consecuencias imprevistas de algunas formas de desarrollo económico. (A/42/427, cap. 1, I, \$9)

Las razones que justificaban ese catastrofismo eran ya sobradamente conocidas entonces: el efecto invernadero, el deterioro de la capa atmosférica de ozono, los contaminantes en el aire, la acidificación del medio ambiente, los desechos tóxicos, la desertificación, la pérdida de los bosques y otras reservas naturales.

Todos estos efectos (A/42/427, cap. 1, I. 3, \$\$ 23-31) llevaron a los redactores de ese Informe a plantear un nuevo enfoque para el medio ambiente y el desarrollo, expresado en los siguientes términos:

El desarrollo no puede subsistir sobre una base de recursos deteriorada ambientalmente; el medio ambiente no puede protegerse cuando el crecimiento deja fuera de cuenta los costos de la destrucción ambiental. Estos problemas no pueden tratarse por separado mediante instituciones y políticas fragmentadas. Están ligados en un complejo sistema de causa a efecto. (A/42/427, cap. 1, II, $\$ 40)$

En definitiva, la propuesta de esta Comisión era la de que "todas las naciones persigan un tipo de desarrollo que integre la producción con la conservación” (A/42/427, cap. 1, II, $\$ 48)$. Pero no solo. Si el principal objetivo del desarrollo es la satisfacción 
de las necesidades y aspiraciones a una vida mejor - como se afirma explícitamente en el Informe (A/42/427, cap. $2, \mathrm{I}, \mathbb{\$}$ ) —, hacer de este un desarrollo duradero requiere que estos consumos, aunque las necesidades estén determinadas social y culturalmente, permanezcan dentro de los límites de lo ecológicamente posible y sin que esto suponga la explotación de los demás (A/42/427, cap. 2, I, $\$ \$ 5-6)$.

Permanecer dentro de lo ecológicamente posible — como también había establecido la Estrategia para la Conservación de la Naturaleza- conlleva dos procederes distintos, ya se trate de recursos renovables o no renovables. Respecto de los primeros (los recursos renovables), evitar su agotamiento implica mantenerse dentro de los límites que establecen la regeneración y el crecimiento natural. En relación con los segundos (los recursos no renovables), se trataría que respecto de los combustibles fósiles se tenga en cuenta su carácter crítico, para impedir que estas fuentes de energía se agoten antes de encontrar otras; $\mathrm{y}$, respecto de los minerales, promoviendo además su reciclaje (A/42/427, cap. 2, I, $\$ \$ 11-12)$. El desarrollo duradero implica también la conservación de las especies vegetales y animales, así como reducir al mínimo los efectos adversos sobre los bienes de todos (a los que el texto unas veces designa como bienes gratuitos y otras como espacios mundiales comunes), de tal manera que se mantenga la integridad del conjunto de ecosistemas (A/42/427, cap. 2, I, \$\$13-14).

La tarea que demanda el desarrollo duradero es inmensa y por eso requiere del concurso de todos, mediante una ética compartida y una política que tenga en cuenta el interés común. Pero como ni lo uno ni lo otro son posibles debido a la primacía de los ambiciosos intereses de las partes implicadas, entonces, solo cabe, a nivel intraestatal, recurrir a leyes, educación, impuestos y subsidios... (A/42/427, cap. 2, II, $\$ \$ 19-20)$; mientras que, a nivel supraestatal, al no existir una autoridad supranacional que dirima estas controversias, la única vía posible pasa por seguir insistiendo en promover una cooperación internacional para el desarrollo, que incida más en los aspectos equitativos que en los del crecimiento:

El desarrollo duradero no solo implica crecimiento. Requiere un cambio en el contenido del crecimiento de manera que sea menos su consumo de materiales y de energía y sean más equitativos sus efectos. Este cambio es necesario en 
todos los países como parte de un conjunto de medidas destinadas a mantener la existencia del capital ecológico, mejorar la distribución de los ingresos y reducir el grado de vulnerabilidad a las crisis económicas. (A/42/427, cap. 2, III, 2, \$35)

Pero, además, apostar por el desarrollo duradero implica — continúa el Informe"que incorporen variables no económicas, tales como la educación y la salud que se disfrutan por su propio bien, el aire y el agua puros y la protección de la belleza natural" (A/42/427, cap. 2, III, 2, \$39).

En definitiva, todavía por esas fechas, no solo las medidas de erradicación de la pobreza se hacían depender del aumento de la productividad, sino que además se insistía en la idea de que el crecimiento económico y el desarrollo son fundamentales para hacer frente a los problemas de la degradación y la protección del medio ambiente. De ahí sus propuestas de crecimiento para las economías en desarrollo de Asia de un $5 \%$ de la renta nacional, de un $5.5 \%$ para las de América Latina, y de un $6 \%$ para África y Asia Occidental; mientras que la tasa mínima de crecimiento económico para los países industriales debería ser de entre el $3 \%$ y el $4 \%$ para que puedan seguir desempeñando su papel en la expansión de la economía mundial (A/42/427, cap. 2, III, $\$ \$ 30-32)$.

Esta idea del continuado crecimiento económico volvió a reiterarse tras la Conferencia de las Naciones Unidas sobre el Medio Ambiente y el Desarrollo (CNUMAD, también denominada Cumbre para la Tierra), convocada por la Asamblea General mediante la resolución 44/228 del 20 de diciembre de 1988 y celebrada en Río de Janeiro (Brasil) del 3 al 14 de junio de 1992, veinte ańos después de la Conferencia de Estocolmo, con la que compartía preocupaciones similares por el deterioro de nuestro entorno.

Sin embargo, a diferencia de aquella otra, tras este tiempo transcurrido, las pruebas aportadas por el primer Informe de Evaluación del Grupo Intergubernamental de Expertos sobre el Cambio Climático terminado en agosto de 1990 y que sirvieron de base para la negociación de la Convención Marco de las Naciones Unidas sobre el Cambio Climático (CMNUCC), no solo evidenciaron la preocupación por el deterioro del sistema climático, sino también por las aún peores previsiones para la salud de 
los sistemas ecológicos terrestres y acuáticos, vitales para el desarrollo y bienestar de las personas, de seguir al mismo ritmo la emisión de gases de efecto invernadero a la atmósfera.

Con todos estos datos, los Estados Parte entonces reunidos elaboraron un documento declarativo final (compuesto por veintisiete principios en los que se concretaban los derechos y responsabilidades), y un plan de acción mundial para promover el desarrollo sostenible llamado Programa 21 (A/CONF.151/26/Rev. 1).

La Declaración sobre el Medio Ambiente y el Desarrollo ligaba sus soluciones a la Declaración de la Conferencia de las Naciones Unidas sobre el Medio Humano, aprobada en Estocolmo el 16 de junio de 1972, en la que se había llegado a relacionar la degradación ambiental con la idea de desarrollo en un doble sentido: por un lado, que "la mayoría de los problemas ambientales están motivados por el subdesarrollo", pero también que "en los países industrializados, los problemas ambientales están generalmente relacionados con la industrialización y el desarrollo tecnológico” (A/CONF.48/14/Rev 1, 1. ${ }^{\text {a }}$ pte., cap. I, \$4). Respecto de lo primero, la solución pasaba, nuevamente, por favorecer el crecimiento económico de las regiones más pobres; respecto de lo segundo, se seguía confiando la solución al progreso tecnológico y la gestión sobre el medio ambiente.

Importante en esta materia habían sido las respuestas ya aportadas en la Carta Mundial de la Naturaleza y el Informe Brundtland. Pero también, tácitamente, el Programa 21, para promover el desarrollo sostenible, tuvo como otros de sus referentes el segundo proyecto de Estrategia Mundial para la Conservación (EMC-II) publicado conjuntamente por la Unión Internacional para la Conservación Naturaleza (UICN), el Programa de Naciones Unidas para el Medio Ambiente (pNuma) y el Fondo Mundial para la Naturaleza (WwF), en octubre de 1991, y en el que se establecieron los principios para una sociedad sostenible. Al respecto, la nueva estrategia explicitó: "Vivir de forma sostenible depende de la aceptación del deber de buscar la armonía con las demás personas y con la naturaleza” (UICN/PNUMA/WwWF, 1991, cap. 1, p. 8). 
La armonía con las demás personas implica que el desarrollo no se realice a expensas de los demás grupos ni de las generaciones venideras:

Nuestro fin debe ser el de compartir equitativamente los beneficios y costes de la utilización de los recursos y la conservación ambiental entre las diferentes comunidades y grupos de intereses, entre las personas pobres y las acomodadas y entre una generación y las que la seguirán. (UICN/PNUMA/wwF, cap. 1, p. 9)

La armonía con la naturaleza exige una gestión del desarrollo que no amenace la supervivencia de otras especies ni destruya sus hábitats: "El desarrollo basado en la conservación debe abarcar actividades explícitamente destinadas a proteger la estructura, las funciones y la diversidad de los sistemas naturales del mundo, de los que nuestra especie es dependiente enteramente" (UICN/PNUMA/WWF, cap. 1, p. 9). Para ello es necesario mantener aptos los sistemas sustentadores de vida (el clima, el aire, el agua y el suelo), conservar toda la gama de variaciones genéricas dentro de cada especie y la variedad de ecosistemas, velar para que el aprovechamiento de los recursos renovables sea ecológicamente sostenible (UICN/PNUMA/WwF, cap. 1, pp. 9-10).

Pero no todos los recursos de la Tierra son renovables, es decir, no se pueden utilizar de una manera sostenible, pero sí se puede lograr prolongar su utilidad reciclándolos, utilizando una menor cantidad de un recurso para fabricar un producto determinado, o adoptando sustitutos renovables cuando sea posible (UICN/PNUMA/WwFF, cap. 1, p. 10).

El propósito no es otro que el de tratar de mantenerse dentro de la capacidad de carga de la Tierra, y ello exige modificar actitudes (valores) y prácticas (comportamientos) personales que sean incompatibles con una forma de vida sostenible.

Para ello, la difusión de la información mediante sistemas educativos (formales y no formales) es parte importante de este proceso de transición hacia sociedades sostenibles. Pero no basta solo con informar, es necesario también facultar a las personas para que cuiden de su propio medio ambiente. Pero esto, en tanto que no lo puede realizar una sola persona, exige de un programa nacional encaminado al 
logro de la sustentabilidad, así como forjar una alianza mundial regida por una ética del cuidado (UICN/PNUMA/WWF, cap. 1, pp. 11-12).

Sin embargo, ante la lentitud en la aplicación del Programa 21 (principalmente de la sección iv, referente a los compromisos adquiridos en relación con los recursos y mecanismos financieros, la transferencia tecnológica y el fortalecimiento de la capacidad para el desarrollo sostenible), la Asamblea General, a través de las resoluciones 47/190 y 51/181, abrió un periodo extraordinario de sesiones al más alto nivel político de participación para realizar un examen y una evaluación globales de la ejecución del Programa 21.

El documento final salido de aquel período, comúnmente conocido como Cumbre de la Tierra +5, celebrado en Nueva York del 23 al 27 de junio de 1997, aprobó un plan para la ulterior ejecución del Programa 21 (S-19/2). Poco después, en diciembre de 2000, la Asamblea General, mediante la resolución 55/199, "profundamente preocupada por el hecho de que [...] desde la Conferencia de las Naciones Unidas sobre el Medio Humano [...] el medio ambiente y la base de recursos naturales que sirven de apoyo a la vida en la tierra siguen deteriorándose a un ritmo alarmante", decidió organizar una nueva Cumbre para examinar los progresos alcanzados y determinar las esferas en que sería necesario redoblar los esfuerzos para ejecutar el Programa 21, las decisiones orientadas hacia la acción que fuera necesario adoptar, los nuevos problemas y oportunidades, y reafirmase los compromisos políticos y las expresiones de apoyo para el logro de un desarrollo sostenible (A/REs/55/199, $\$ \$$ 1-3) que tomarían nueva expresión en posteriores cumbres mundiales.

La Cumbre Mundial sobre el Desarrollo Sostenible (también conocida como Río +10) se celebró en Johannesburgo (Sudáfrica) del 26 de agosto al 4 de septiembre de 2002. El documento final de la Cumbre (A/CONF.199/20) incluyó una nueva declaración política y un plan de aplicación que insistía en los mismos términos que los anteriores.

Entretanto, el 22 de diciembre de 2001 la Asamblea General, mediante la resolución 66/197, convocaba una Conferencia de las Naciones Unidas sobre el Desarrollo Sostenible que se celebraría nuevamente en Río de Janeiro (Brasil) del 
20 al 22 de junio de 2012, cuyo documento final, El futuro que queremos, mostró la visión común que tenían los jefes de Estado y de Gobierno, y los representantes de alto nivel, y que fue expresada en estos términos:

Reconocemos que la erradicación de la pobreza, la modificación de las modalidades insostenibles y la promoción de modalidades de consumo y producción sostenibles, y la protección y ordenación de la base de recursos naturales del desarrollo económico y social son objetivos generales y requisitos indispensables del desarrollo sostenible. Reafirmamos también que es necesario lograr el desarrollo sostenible promoviendo un crecimiento sostenido, inclusivo y equitativo, creando mayores oportunidades para todos, reduciendo las desigualdades, mejorando los niveles de vida básicos, fomentando el desarrollo social equitativo y la inclusión, y promoviendo la ordenación integrada y sostenible de los recursos naturales y los ecosistemas, que contribuye, entre otras cosas, al desarrollo económico, social y humano y facilita al mismo tiempo la conservación, la regeneración, el restablecimiento y la resiliencia de los ecosistemas frente a los problemas nuevos y en ciernes. (A/CONF.216/16, an., I, \$4)

El reconocimiento así expresado — síntesis de los planteamientos sobre el desarrollo sostenible - los llevó a reafirmar su compromiso con el fortalecimiento de la cooperación internacional, una de cuyas últimas expresiones fue la Declaración del Milenio (Resolución 55/2) y sus objetivos marcados para el año 2015 (A/54/2000, pte. III, A-G, $\$ \$ 76-188$ y pte. vII, $\$ 364)$.

Al final de este período, el informe presentado por Naciones Unidas mostraba — presupuesta la veracidad de este- un panorama muy distinto al de períodos anteriores: reducción de la pobreza extrema y el porcentaje de personas con nutrición insuficiente, mejoras habitacionales y en los servicios públicos, mayores índices de escolaridad y menores tasas de disparidad entre géneros, menor índice de mortandad materna e infantil, mejora en la prevención y atención sanitaria, etc. 
Pero de igual manera que hay que poner de relieve la consecución de todas aquellas metas, también conviene constatar el enorme retroceso en la incorporación de los principios de la conservación medioambiental en las políticas y los programas nacionales.

Cierto es que, hasta la fecha, gracias al cumplimiento del Protocolo de Montreal (A/RES/49/114), prácticamente se habían eliminado las sustancias que agotan la capa de ozono - y se estima que esta se recupere a mediados del siglo XxI- No es menos cierto que, gracias a diversos acuerdos, las áreas terrestres y marítimas protegidas han aumentado considerablemente en las últimas décadas, cubriendo actualmente un $15.4 \%$ de la superficie terrestre del planeta, incluyendo las aguas interiores. Pero hay muchos otros aspectos de la degradación ambiental que socavan las posibilidades de un desarrollo sostenible: la sobreexplotación de las poblaciones de peces marinos, la pérdida de hectáreas de bosque (y la biodiversidad que alberga) y el acrecentamiento de las emisiones de dióxido de carbono a la atmósfera (que se han incrementado en más de un $50 \%$ desde 1990), con los efectos perjudiciales que este aumento comporta sobre ecosistemas y hábitats, así como respecto a la disponibilidad de agua dulce y alimento que resulta de la elevación de la temperatura media del planeta, que pone en seria cuestión los logros del desarrollo y sus nuevas ambiciosas metas adoptadas por unanimidad el 25 de septiembre de 2015, durante el sexagésimo noveno periodo de sesiones de Asamblea General de las Naciones Unidas (Resolución A 70-L1).

La razón es sencilla. Basta con recordar que el objetivo 8.1 de Naciones Unidas para el logro del desarrollo sostenible es promover el crecimiento económico sostenido (Resolución A 70-L1, $\$$ 59). También en la Conferencia de Desarrollo Sostenible Río +20 , en el documento El futuro que queremos, se reafirmó en su propósito de leemos- "promover el crecimiento económico sostenido e inclusivo, el desarrollo social y la protección del medio ambiente" (A/RES/66/288, I, $\$ \$ 2-6$ ), lo cual se estimaba que redundara en beneficio de todos.

Como se comprueba, el cambio es sustancial: si originariamente la preocupación era hacer frente a la realidad de lo limitado que son los recursos y la capacidad de los ecosistemas para respaldar la vida de la presente y las futuras generaciones, el nuevo 
propósito es promover un crecimiento económico sostenido; con lo que no puede ser más diferente el uso dado al término desde que la Comisión Brundtland insistiera en la idea de crecimiento económico como medio para mejorar las condiciones de vida de millones de personas desfavorecidas. El desarrollo sostenible exige que se satisfagan las necesidades básicas de todos y que se extienda a todos la oportunidad de colmar sus aspiraciones a una vida mejor $(\mathrm{A} / 42 / 427, \mathrm{I}, 3, \S 27)$.

Tanto lo uno (satisfacer las necesidades básicas de los pobres) como lo otro (pretender niveles mayores de satisfacción) requiere, asílo reconocía el último de los documentos, "no solo una nueva era de crecimiento económico para las naciones donde los pobres constituyen la mayoría, sino la garantía de que estos pobres recibirán la parte que les corresponde de los recursos necesarios para sostener ese crecimiento" (A/42/427, I, $3, \$ 28)$. Sin embargo, aquellas segundas — respecto del reparto equitativo de la riqueza a nivel global - no encontraron su solución con los planes de desarrollo internacionales. Por el contrario, a nivel social ha seguido ampliándose la brecha entre unos países y otros, no solo en términos de riqueza cuantificada en índices como el producto interno bruto (PIB), sino también respecto a la degradación ambiental, como indicaba en 2014 el Informe Planeta Vivo (figura 1).

Figura 1. Carga de los países según ingresos sobre la biocapacidad mundial

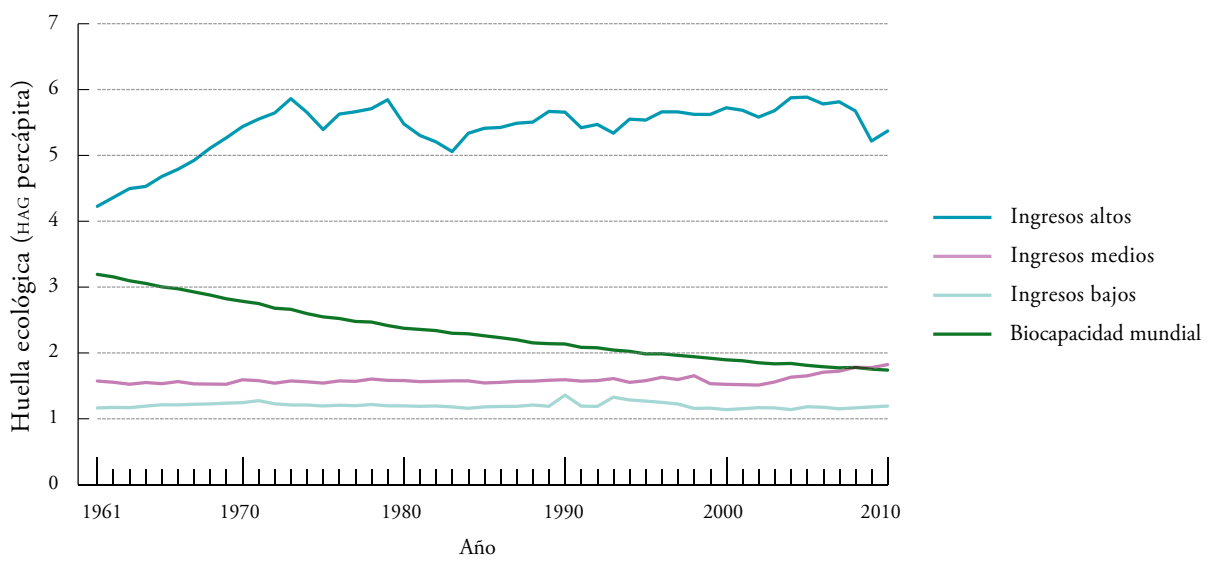

Fuente: tomada de IPV (2014). 


\section{CONCLUSIÓN: LA CRÍTICA DEL IMAGINARIO SOCIAL DESARROLLISTA}

Si el uso verborreico de la expresión desarrollo sostenible nos ha conducido a tamaño problema socioambiental, entonces buena parte de la solución estará en un manejo conceptual apropiado; pero no solo esto, porque además de esta reconsideración acerca de los continuados propósitos del desarrollo estaría el hecho de que revertir la actual deriva de deterioro, ha de comportar no solo una seria crítica a la sociedad de consumo de masas, sino al imaginario social que la sostiene.

Pero mientras que la idea de romper con el imaginario desarrollista en los países enriquecidos implica una apuesta que se decida por un cambio de valores, hábitos de vida, relaciones sociales de producción, de reparto y de distribución de las riquezas (Latouche, 2009b, p. 167), de modo tal que contribuyan a disminuir la presión sobre la biósfera y restaurar un mínimo de justicia social (Latouche, 2009a, p. 17); en los países empobrecidos comportará además un intento de descolonización del saber (Latouche, 2009a, p. 12) que, al tiempo que ayude a desmitificar el desarrollismo y la occidentalización del mundo (Latouche, 2009d, p. 22), sea capaz de reestablecer el hilo de su historia roto por la colonización y el imperialismo (Latouche, 2009a, p. 19; 2009c, pp. 76-77).

Pero esto último, si bien parece más fácil de resolver en cuestiones de ejercicio de poder y su legitimación, no lo es en el ámbito del saber; pues, para ello, no basta con señalar los problemas devenidos, sino que cualesquiera soluciones que se pretendan disyuntivas requieren una revisión a fondo de los presupuestos que promueven la idea de modernidad, que, al ser de carácter enteramente filosóficos, requeriría que nos entrometiéramos en hondas consideraciones que han conformado nuestro actual paradigma epistemológico. Unos comedimientos que requieren, previamente, atinar con las verdaderas cuestiones de fondo; lo cual no parece haberse emprendido con seriedad y, por lo tanto, ambos propósitos, aquí tan solo apuntados, no son capaces de articular alternativa alguna a la actual deriva desarrollista y sus devastadoras secuelas, tan solo añadir mayor confusión a las distintas problemáticas, persistiendo así todos los malestares que ellas provocan.

Sin embargo, aun tratándose de una cuestión fundamental, los márgenes de este ensayo no permiten mayores alargamientos al respecto, teniéndonos que conformar 
únicamente con haber señalado las contradicciones a partir de las cuales comenzar a pensar. Para lo cual quizá podamos plantearnos unas pocas cuestiones. A saber: ¿En verdad hallaríamos una solución a nuestra deteriorada situación reabriendo debates historicistas? ¿Nos beneficia para nuestro asunto volver sobre discusiones epistemológicas tardomedievales para repensar nuestro sentido del progreso? Y, en definitiva, ¿requiere la actual crisis socioambiental un nuevo modelo de ética, diferente en sistematización, método y contenidos? Si a estas interrogaciones las respuestas fueran negativas, ya tenemos buena parte de la solución: hagámonos simples para apremiar las cautelas, dejándonos de consideraciones abstrusas y entretenimientos academicistas pedantes que alargan auténticos pesares o, de lo contrario, cada vez encontraremos menos motivos para el mantenimiento de la paz entre los pueblos y sus gentes; pues, sabido es que si lo primerísimo es el bien de las personas, todo amparo debe enfocarse en respetar, preservar y salvaguardar las condiciones que faciliten la satisfacción de las necesidades y el fomento de las capacidades, tal como se hallan concebidos por cada sociedad.

Lo contrario seguirá siendo susceptible de padecer distintos tipos de hostilidades: unas como resultado de la dificultad — cuando no de la imposibilidad- de acceder a recursos naturales en condiciones adecuadas en calidad y cantidad, debido a impedimentos impuestos por las actividades abusivas de algunos agentes intencionados; otras devenidas de la imposibilidad de satisfacer los mínimos vitales en un mundo en el que - como ilustramos mediante la figura 1- la posesión de las riquezas naturales está desigualmente distribuida y desproporcionadamente acopiada; pero también como resultado de la progresiva pérdida de la propia identidad que, hasta la fecha, ha favorecido el seguimiento obligado de una sola visión sobre el desarrollo, a pesar de los pesares.

Estos son tres posibles ámbitos de actuación a los que, a todos, individual y solidariamente, nos urge atender tanto para bien de los presentes como provecho de los venideros. De lo contrario, en medio de esta paz imperfecta, inacabada, aplazada en muchos aspectos, como los apuntados a lo largo de este escrito, y por lo tanto inconclusa, como es la que actualmente regula nuestro marco global, no habrá de extrañar que, a la postre, tamaños menosprecios acaben evidenciándose insostenibles para dar cumplida cuenta siquiera de aquello que en el preámbulo de la Declaración 
Universal de los Derechos Humanos (Resolución 217 A [III]) se expresara como una manera de promover — literalmente— las relaciones amistosas, pues tales siguen siendo los agravios e inconvenientes que persisten tras la complaciente idea del desarrollo a la que se adjetiva como sostenible, a pesar de los muchos desórdenes socioambientales que, como hemos visto, comporta.

\section{REFERENCIAS}

Coen Anitúa, A. (2006), De sostenible y sustentable. Sentidos y significados. Correo del Maestro, (116), 50-51.

Costanza, R. y Patten, B. C. (1995), Defining and predicting sustainability. Ecological Economics, 15(3), 193-196.

Latouche, S. (1989). L'Occidentalisation du monde. Essai sur la signification, la portée et les limites de l'uniformisation planétaire. Paris: La Découverte/Poche.

Latouche, S. (1993). El planeta de los náufragos: Ensayo sobre el posdesarrollo. Madrid: Acento.

Latouche, S. (2009a). Decrecimiento y posdesarrollo: el pensamiento creativo contra la economía del absurdo. Barcelona: El Viejo Topo.

Latouche, S. (2009b). La apuesta por el decrecimiento: ¿cómo salir del imaginario dominante? Barcelona: Icaria.

Latouche, S. (2009c). Pequeño tratado del decrecimiento sereno. Barcelona: Icaria.

Latouche, S. (2009d). Sobrevivir al desarrollo: de la descolonización del imaginario económico a la construcción de una sociedad alternativa. Barcelona: Icaria.

Latouche, S. (2012). La sociedad de la abundancia frugal: contrasentidos y controversias del decrecimiento. Barcelona: Icaria. 
Latouche, S. (2014a). Hecho para tirar: la irracionalidad de la obsolescencia programada. Barcelona: Octaedro.

Latouche, S. (2014b). Limite. Buenos Aires: Adriana Hidalgo.

Naciones Unidas (NN. uU.). (1945). 1 unTs xvi. Carta de las Naciones Unidas. Nueva York: Naciones Unidas.

Naciones Unidas (NN. UU.). (1948). Documento A/RES/198(III). Desarrollo económico de los paises insuficientemente desarrollados. Nueva York: Naciones Unidas.

Naciones Unidas (NN. UU.). (1960). Resolución 1514(xv). Declaración sobre la concesión de la independencia a los paises y pueblos coloniales. Nueva York: Naciones Unidas.

Naciones Unidas (nN. UU.). (1961a). Documento A/Res/1715(xvi). Decenio de las Naciones Unidas para el Desarrollo. Nueva York: Naciones Unidas.

Naciones Unidas (nN. uU.). (1961b). Documento A/REs/1710(xvi). Decenio de las Naciones Unidas para el Desarrollo. Programa de Cooperación Económica Internacional. Nueva York: Naciones Unidas.

Naciones Unidas (nN. UU.). (1963). Resolución 1904 (XVIII). Declaración de las Naciones Unidas sobre la Eliminación de todas las Formas de Discriminación Racial. Nueva York: Naciones Unidas.

Naciones Unidas (NN. uU.). (1965). Resolución 2106 A (xx). Convención Internacional sobre la Eliminación de todas las Formas de Discriminación Racial. Nueva York: Naciones Unidas.

Naciones Unidas (nn. uU.). (1968). Documento A/RES/2398(xxiII). Problemas del Medio Humano. Nueva York: Naciones Unidas.

Naciones Unidas (NN. UU.). (1969). Documento A/RES/2542(xxIV). Declaración sobre el Progreso y el Desarrollo en lo Social. Nueva York: Naciones Unidas. 
Naciones Unidas (NN. UU.). (1970). Documento A/Res/2626 (xxv). Estrategia Internacional del Desarrollo para el Segundo Decenio de las Naciones Unidas para el Desarrollo. Nueva York: Naciones Unidas.

Naciones Unidas (NN. UU.). (1971). Documento A/REs/2626(xxv). Estrategia Internacional del Desarrollo para el Segundo Decenio de las Naciones Unidas para el Desarrollo. Nueva York: Naciones Unidas.

Naciones Unidas (NN. uU.). (1972). Documento A/Conf.48/14/Rev.1. Informe de la Conferencia de las Naciones Unidas sobre el Medio Humano. Nueva York: Naciones Unidas.

Naciones Unidas (nn. uU.). (1974a). Resolución 3281 (xxix). Carta de Derechos y Deberes Económicos de los Estados. Nueva York: Naciones Unidas.

Naciones Unidas (Nn. uU.). (1974b). Documento a/Res/3202(S-VI). Programa de acción sobre el establecimiento de un nuevo orden económico internacional. Nueva York: Naciones Unidas.

Naciones Unidas (NN. UU.). (1974c). Documento A/REs/3201(S-VI). Declaración sobre el establecimiento de un nuevo orden económico internacional. Nueva York: Naciones Unidas.

Naciones Unidas (NN. UU.). (1975). Documento A/Res/3362(S-VII). Desarrollo y cooperación económica internacional. Nueva York: Naciones Unidas.

Naciones Unidas (NN. UU.). (1980). Documento a/res/35/56. Estrategia Internacional del Desarrollo para el Tercer Decenio de las Naciones Unidas para el Desarrollo. Nueva York: Naciones Unidas.

Naciones Unidas (NN. Uu.). (1986a). Documento A/REs/41/128. Declaración sobre el derecho al desarrollo. Nueva York: Naciones Unidas.

Naciones Unidas (NN. UU.). (1986b). Documento A/REs/37/7. Carta Mundial de la Naturaleza. Nueva York: Naciones Unidas. 
Naciones Unidas (NN. uU.). (1987). Documento A/42/427. Informe de la Comisión Mundial sobre el Medio Ambiente y el Desarrollo, "Nuestro futuro común”. Nueva York: Naciones Unidas.

Naciones Unidas (NN. UU.). (1990). Documento A/RES/45/199. Estrategia Internacional del Desarrollo para el Cuarto Decenio de las Naciones Unidas para el Desarrollo. Nueva York: Naciones Unidas.

Naciones Unidas (NN. uU.). (1992). Treaty Series, 1760(30619). New York: Naciones Unidas.

Naciones Unidas (NN. UU.). (1992). Documento A/CONF.151/26 (vols. I a IV). Informe de la Conferencia de las Naciones Unidas sobre el Medio Ambiente y el Desarrollo. Nueva York: Naciones Unidas.

Naciones Unidas (nN. uU.). (1994). Documento a/Conf.167/9. Informe de la Conferencia Mundial sobre el Desarrollo Sostenible de los Pequeños Estados Insulares en Desarrollo. Nueva York: Naciones Unidas.

Naciones Unidas (NN. UU.). (1994). Documento A/CONF.171/13/Rev.1. Informe de la Conferencia Internacional sobre la Población y el Desarrollo. Nueva York: Naciones Unidas.

Naciones Unidas (NN. uU.). (1994). Treaty Series, 1771(30822). New York: Naciones Unidas.

Naciones Unidas (NN. UU.). (1995). Documento A/COnf.177/20. Informe de la cuarta Conferencia Mundial sobre la mujer. Nueva York: Naciones Unidas.

Naciones Unidas (nN. UU.). (1997). Documento A/Res/51/240. Programa de Desarrollo. Nueva York: Naciones Unidas.

Naciones Unidas (NN. UU.). (1997). Resolución S-19/2. Examen y evaluación generales de la ejecución del Programa 21. Nueva York: Naciones Unidas.

Naciones Unidas (NN. UU.). (1999). Resolución S-21/2. Medidas clave para seguir ejecutando el Programa de Acción de la Conferencia Internacional sobre la Población y el Desarrollo. Nueva York: Naciones Unidas. 
Naciones Unidas (NN. UU.). (1999). Treaty Series, 1954(33480). New York: Naciones Unidas.

Naciones Unidas (NN. UU.). (2000a) Documento A/54/2000. Nosotros los pueblos: la función de las Naciones Unidas en el siglo XXI. Nueva York: Naciones Unidas.

Naciones Unidas (NN. UU.). (2000b) Resolución 55/2. Declaración del milenio. Nueva York: Naciones Unidas.

Naciones Unidas (NN. uU.). (2000c). Documento A/REs/55/199. Examen decenal de los progresos logrados en la aplicación de los resultados de la Conferencia de las Naciones Unidas sobre el Medio Ambiente y el Desarrollo. Nueva York: Naciones Unidas.

Naciones Unidas (NN. uU.). (2001). Documento a/Conf.189/12. Informe de la Conferencia Mundial contra el Racismo, la Discriminación Racial, la Xenofobia y las Formas Conexas de Intolerancia. Nueva York: Naciones Unidas.

Naciones Unidas (nN. uU.). (2002a). Documento A/Conf.199/20. Informe de la Cumbre Mundial sobre el Desarrollo Sostenible. Nueva York: Naciones Unidas.

Naciones Unidas (NN. uU.). (2002b). Documento A/Conf.198/11. Informe de la Conferencia Internacional sobre la Financiación para el Desarrollo. Nueva York: Naciones Unidas.

Naciones Unidas (NN. uU.). (2002c). Documento A/57/304. Examen y evaluación finales de la aplicación del Nuevo Programa de las Naciones Unidas para el Desarrollo de África en el Decenio de 1990. Nueva York: Naciones Unidas.

Naciones Unidas (NN. uU.). (2003) Documento A/Conf.202/3. Informe de la Conferencia Ministerial Internacional de Países en Desarrollo sin Litoral y de Tránsito y de Países Donantes y de las Instituciones Financieras y de Desarrollo Internacionales sobre la Cooperación en materia de Transporte de Tránsito. Nueva York: Naciones Unidas.

Naciones Unidas (NN. UU.). (2005a) Documento A/Conf.207/11. Informe de la Reunión Internacional para examinar la ejecución del Programa de Acción para el desarrollo sostenible de los pequeños Estados insulares en desarrollo. Nueva York: Naciones Unidas. 
en Ciencias Sociales

Naciones Unidas (NN. uU.). (2005b) Resolución 60/1. Documento Final de la Cumbre Mundial 2005. Nueva York: Naciones Unidas.

Naciones Unidas (NN. uU.). (2008a). Resolución 63/239. Declaración de Doha sobre la financiación para el desarrollo: documento final de la Conferencia internacional de seguimiento sobre la financiación para el desarrollo encargada de examinar la aplicación del Consenso de Monterrey. Nueva York: Naciones Unidas.

Naciones Unidas (NN. UU.). (2008b). Resolución 63/1. Declaración política sobre las necesidades de África en materia de desarrollo. Nueva York: Naciones Unidas.

Naciones Unidas (NN. uU.). (2011) Documento a/Conf.219/7. Informe de la Cuarta Conferencia de las Naciones Unidas sobre los Paises Menos Adelantados. Nueva York: Naciones Unidas.

Naciones Unidas (nN. uU.). (2012a). Documento A/Conf.216/16. Informe de la Conferencia de las Naciones Unidas sobre el Desarrollo Sostenible. Nueva York: Naciones Unidas.

Naciones Unidas (NN. UU.). (2012b). Documento A/REs/66/288. El futuro que queremos. Nueva York: Naciones Unidas.

Naciones Unidas (NN. UU.). (2015). Documento A/70/L.1. Transformar nuestro mundo: la Agenda 2030 para el Desarrollo Sostenible. Nueva York: Naciones Unidas.

Naciones Unidas (nN. uu.). (2015). Objetivos de Desarrollo del Milenio: Informe de 2015. Nueva York: Naciones Unidas.

Pigou, A. C. (2016). La economía del bienestar (vol. 2). Navarra: Thomson Reuters.

UICN. (I980). Estrategia Mundial para la Conservación de la Naturaleza. La conservación de los recursos vivos para el logro de un desarrollo sostenido. Gland: UICN.

UICN. (I99I). Cuidar la Tierra: estrategia para el futuro de la vida. Gland: UICN. 
UICN. (2006): "El futuro de la sostenibilidad. Repensando el Medio Ambiente y el Desarrollo en el siglo Veintiuno". Gland: UICN.

World Wide Fund for Nature (wwF). (2006). Informe Planeta Vivo 2006. Gland: wwF.

World Wide Fund for Nature (wwF). (2008). Informe Planeta Vivo 2008. Por un planeta vivo. Gland: WwF.

World Wide Fund for Nature (wwF). (2010). Informe Planeta Vivo 2010. Biodiversidad, biocapacidad y desarrollo. Gland: WwF.

World Wide Fund for Nature (WwF). (2012). Informe Planeta Vivo 2012. Biocapacidad, biodiversidad y propuestas de futuro. Gland: wwF.

World Wide Fund for Nature (wwF). (2014). Informe Planeta Vivo 2014. Resumen. Gland: WWF.

World Wide Fund for Nature (wwF). (2016). Informe Planeta Vivo 2016. Riesgo y resiliencia en la nueva era. Gland: wwF.

World Wide Fund for Nature (wwF). (2018). Informe Planeta Vivo-2018: Apuntando más alto. Gland: wwF. 